\title{
The Role of Fiberoptic bronchoscopy in Evaluating The causes of Undiagnosed Pleural Effusion
}

\author{
Anbananthan krishnasamy ${ }^{1}$, Keerthivasan Sivanmani ${ }^{2}$ \\ ${ }^{\text {I}}$ (Department of TB\& Chest Diseases, Coimbatore Medical College, \\ The Tamilnadudr.MGR Medical University, India.) \\ ${ }_{2}^{2}$ (Department of TB\& Chest Diseases, Coimbatore Medical College, \\ The Tamilnadudr.MGR Medical University, India.)
}

\begin{abstract}
Background and objectives:

To evaluate the diagnostic merits of Fiberoptic bronchoscopy in evaluating the causes of undiagnosed pleural effusion.

Methods:

This is a Prospective(Observational) study, conducted in the Department of Thoracic Medicine, Coimbatore Medical College, Coimbatore for a period of one year from June 2015 to June 2016. A total of 110 patients were included in this study. All eligible and consenting patients were subjected to routine blood investigations, sputum for Acid Fast Bacilli, chest skiagram, mantoux test and pleural fluid analysis, Pleural biopsy and Fiberoptic bronchoscopy were done and specimens were sent for microbiological, cytologicaland his to pathological analysis.

Results:

Among 110 patients with pleural effusion, after initial work up, diagnosis was made in 43(39.09\%) patients. In the remaining 67 patients whose diagnosis was not made by initial workup, 3 patients were not willing for bronchoscopy and pleural biopsy. Out of 64 patients, Fiberoptic Bronchoscopy was useful in making diagnosis in $18(28.1 \%)$ patients. Pleural biopsy helped in diagnosing 26(40.62\%) patients.

Conclusion:

Fiberoptic bronchoscopy is useful in patients with exudative effusion still un diagnosed after pleural fluid cytologyand biopsy who presents with parenchymal abnormalities on chest skiagram or with hemoptysis.
\end{abstract}

Keywords:

Acid Fast Bacilli, Exudative pleural effusion, Fiberoptic bronchoscopy, pleural biopsy.

\section{Introduction}

Determining the cause of a pleural effusion is greatly facilitated by analysis of the pleural fluid. Thoracentesis is a simple bed side procedure that permits fluid to be rapidly sampled, visualized, examined microscopically and quantified. A systematic approach to analysis of the fluid in conjunction with the clinical presentation should allow the clinician to diagnose the cause of an effusion. A definitive diagnosis is provided by the finding of malignant cells or specific organisms in the pleural fluid can be established in approximately $25 \%$ of patients. Pleural effusion remain sun diagnosed after routine tests in pleural fluid in many patients. So, we need a simple and safe investigative tool to evaluate undiagnosed effusion. This study is designed to diagnose the cause so fun diagnosed effusions by using Fiberoptic bronchoscope, where pleural fluid analysis and closed pleural biopsy were in conclusive.

\section{Materials and Methods}

A Prospective (Observational) study was carried out in Department of Thoracic Medicine for one year. Patients with the diagnosis of pleural effusion, fulfilling inclusion criteria were admitted in Thoracic Medicine Ward at Coimbatore Medical College Hospital. Inclusion criteria were individuals of more than 14 years of age with diagnosis of pleural effusion. Routine blood investigations, Chest skiagram, Sputum for Acid Fast Bacillus, Mantoux test and Pleural fluid analysis were done for all patients. Pleural fluid was sent for bio chemical, microbiological, and cytological analysis. Informed and written consents were obtained from the patient. For patients with exudative Pleural effusion to whom initial investigations were in conclusive, pleural biopsy and Fiberoptic bronchoscopy were done under strict aseptic precaution under local anaesthesia and specimen were sent for biochemical, microbiological, cytological and histopathological analysis as required. 


\section{Results}

We included 110 pleural effusion patients in our study. With initial workup, diagnosis was made in 43(39.09\%) patients. Transudative effusion was most commonly diagnosed in 20 patients, followed by tuberculosis (sputumAFB+ve) in 10 patients. Cytology positive for malignant cells and parapneumonic effusion each seen in 5 patients. Pancreatitis was diagnosed in 2 patients and Lympho main one patient. Out of 67 patients in whom initial work up was in conclusive, 3 patients were not willing for Fiberoptic Bronchoscopy and Pleural biopsy procedure. Pleural biopsy was done in 54 patients after obtaining informed written consent. Pleural biopsy was diagnostic in 26(40.62\%) patients. Most common diagnosis made was Tuberculosis in 18 patients followed by Adeno carcinoma in 5 patients, and then by Metastatic carcinoma, Squamo us cell carcinoma, and Small cell carcinoma each in one patient.

FOB was done in rest of 64 patients after obtaining informed written on sent. Findings observed in bronchoscopy were Erythema, Nodularity/ Sessile lesion, Polypoidallesion, External compression. FOB was normal in 25 patients. Erythema was seen in 5 patients, but none of them show positive results. Nodularity / Sessile lesion was seen in 16 patients, out of them 10 patients showed positive results. In those 10 patients, 9 were diagnosed as Malignancy and 1 as Endobronchial Tuberculosis. Polypoidal lesion was seen in 5 patients, all of them showed positive results as Malignancy. External compression was seen in 13 patients, out of them 3 patients showed positive results as Endobronchial Tuberculosis. Totally, FOB was able to diagnose 18 cases (28.1\%) out of 64 patients in whom initial work up was in conclusive. Out of 18 cases that were diagnosed from FOB, 14 cases diagnosed as Malignancy and 4 cases as Endobronchial Tuberculosis. In Malignancy, Squamous cell carcinoma was most commonly diagnosed in 6 cases, followed by Small cell carcinoma in 5 cases, and then by Adenocarcinoma in 3 cases.

We analyzed the study patients in whom initial work up was in conclusive, based on clinical history of hemoptysis. Hemoptysis was present in 26 patients and absent in 38 patients. The yield from Bronchoscopy was more in patients with hemoptysis. The yield from Pleural fluid examination was more in patients who had no hemoptysis.

Apart from hemoptysis we also analyzed the study patients based on radiologic pattern, as those who presented only with pleural effusion in 42 patients, and those with pleural effusion and parenchymal abnormality in 22 patients. The yield from Bronchoscopy was more in patients who presented with both Pleural effusion and Parenchymal abnormality. The yield from Pleural fluid examination was more in patients who presented only with Pleural effusion.

IV. Figures And Tables

Table 1. Diagnosis after initial work up

\begin{tabular}{|c|c|}
\hline Diagnosis & No of cases \\
\hline Transudative effusion & 20 \\
\hline Sputum AFB +ve & 10 \\
\hline Cytology +ve for malignant cells & 5 \\
\hline Lymphoma & 1 \\
\hline Pancreatitis & 2 \\
\hline Para pneumonic effusion & 5 \\
\hline Total & $\mathbf{4 3 ( 3 9 . 0 9 \% )}$ \\
\hline
\end{tabular}




\section{Diagnosis after Initial work up}

Transudative effusion

Sputum AFB +ve

- Cytology +ve for malignant cells a Lymphoma

Pancreatitis

Parapneumonic effusion

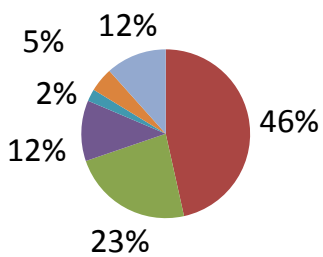

Table 2. Pleural biopsy

\begin{tabular}{|c|c|}
\hline Diagnosis & Number \\
\hline Tuberculosis & 18 \\
\hline Metastatic carcinoma & 1 \\
\hline Adenocarcinoma & 5 \\
\hline Squamous cell carcinoma & 1 \\
\hline Small cell carcinoma & 1 \\
\hline Total & $\mathbf{2 6 ( 4 0 . 6 2 \% )}$ \\
\hline
\end{tabular}

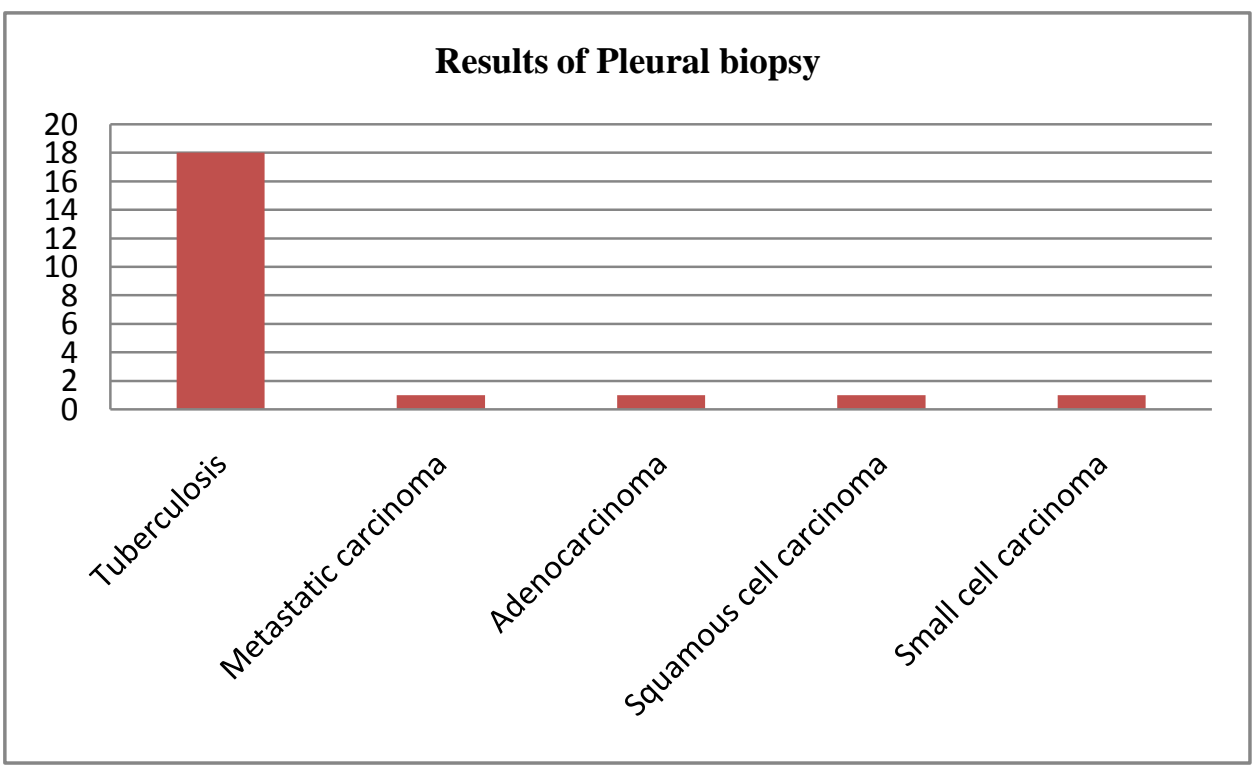

Table3. Bronchoscopy Results

\begin{tabular}{|c|c|c|}
\hline Findings & Number & Positiveresults \\
\hline Normal & 25 & 0 \\
\hline Erythema & 5 & 10 \\
\hline Nodularity /esessile lesion & 16 & 5 \\
\hline Polypoidal growth & 5 & 3 \\
\hline External compression & 13 & $\mathbf{1 8}(\mathbf{2 8 . 1 \%})$ \\
\hline Total & $\mathbf{6 4}$ & 0 \\
\hline
\end{tabular}




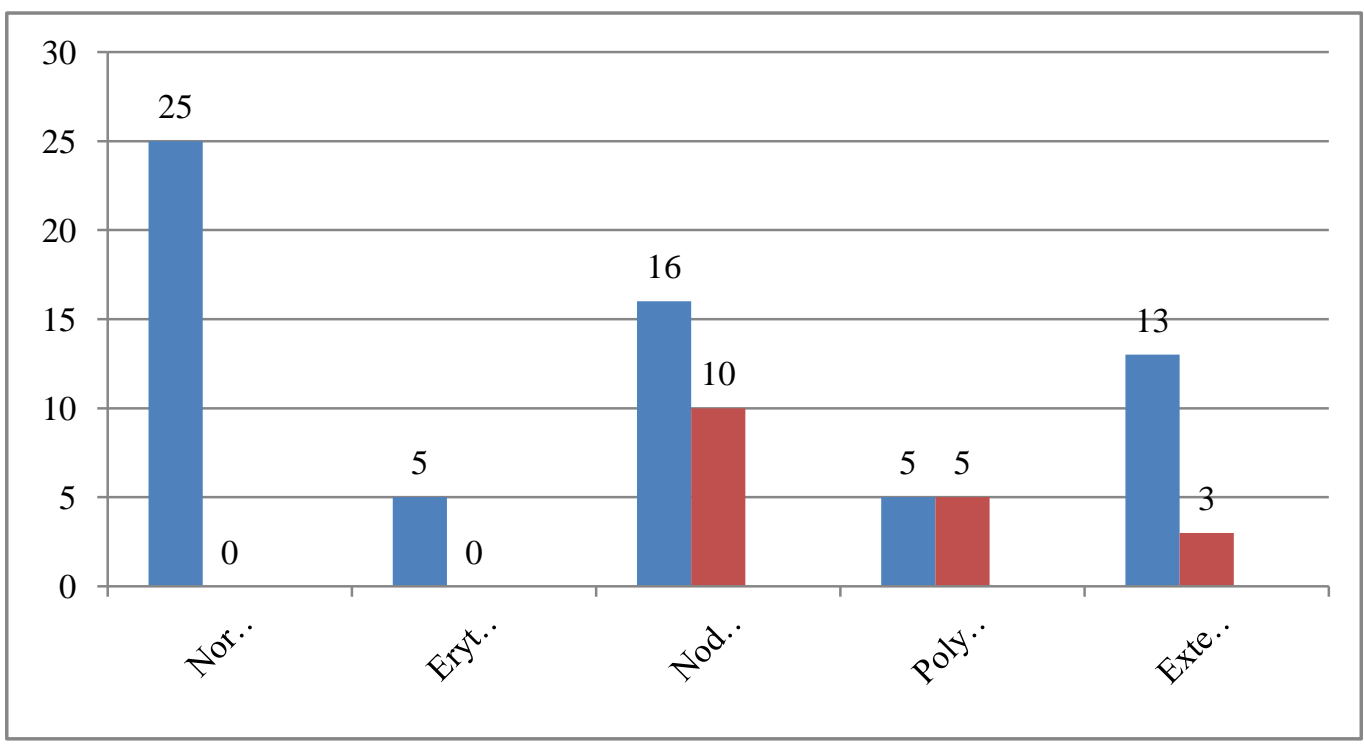

Table 4.Results of Bronchoscopy and Pleural fluid examination in patients classified by hemoptysis.

\begin{tabular}{|c|c|c|c|c|l|}
\hline Hemoptysis & No & $\begin{array}{c}\text { Br.Wash (or) } \\
\text { Biospy }\end{array}$ & $\begin{array}{c}\text { Pleural fluid } \\
\text { examination }\end{array}$ & P-Value & Test \\
\hline Present & 26 & 13 & 8 & $0.000<0.05$ & \\
\hline Absent & 38 & 5 & 18 & $0.000<0.05$ & tudent t test \\
\hline
\end{tabular}

Table-5 Results OF Bronchoscopy and Pleural fluid examination in patients classified by Radiological pattern

\begin{tabular}{|c|c|c|c|c|c|}
\hline $\begin{array}{c}\text { Radiologic } \\
\text { Pattern }\end{array}$ & No & $\begin{array}{c}\text { Br.Wash (or) } \\
\text { Biospy }\end{array}$ & $\begin{array}{c}\text { Pleuralfluid } \\
\text { examination }\end{array}$ & P- Value & Test \\
\hline Only Pleural effusion & 42 & 4 & 19 & $0.000<0.05$ & \\
\hline $\begin{array}{c}\text { Pleural Effusion+ } \\
\text { Parenchymal } \\
\text { Abnormality }\end{array}$ & 22 & 14 & 7 & $0.000<0.05$ & $\begin{array}{c}\text { Student } \mathrm{t} \\
\text { Test }\end{array}$ \\
\hline
\end{tabular}

\section{Discussion}

In 19 to $25 \%$ of patients, the cause of pleural effusion remains unexplained after pleural fluid analysis and pleural biopsy. Fiberoptic Bronchoscopy is a useful tool in these patients of unexplained pleural effusion. Chang et al performed Bronchoscopy, Thoracentesis and Pleural biopsy on 140 consecutive patients with pleural effusion. In the patient group with an isolated pleural effusion, with no hemoptysis or pulmonary abnormality on the chest radiograph, the yield from bronchoscopy was $16 \%$ where as pleural investigation yielded a positive diagnosis in $61 \%$. In the patient group of pleural effusion with hemoptysis or pulmonary abnormality, the yield from bronchoscopy was more than $70 \%$ where as the yield from pleural investigation was less than $35 \%$. Williams et al evaluated the role of FOB in 28 patients with pleural effusion of undetermined etiology. In this group,4 patients had a diagnostic FOB,3 for malignancy and one for tuberculosis. They concluded that FOB was of value in the evaluation of patients with undiagnosed pleural effusion. In our study, Out of 64 patients whose diagnosis was not made by initial workup, FOB was useful in making diagnosis in $18(28.1 \%)$ cases ( $\mathrm{p}$ value-0.000<0.005). Pleural biopsy helped in diagnosing $26(40.62 \%)$ cases (p value- $0.000<0.005)$. Pleural biopsy is particularly important for diagnosing tuberculosis but will also slightly increase the yield for malignancy. For patients with exudative effusion still undiagnosed after pleural fluid examination and with parenchymal abnormalities on chest radiograph or with hemoptysis, Fiberoptic bronchoscopy is a useful next step. When combining Initial work up, Pleural biopsy and Fiberoptic Bronchoscopy in our study, 87 patients $(79.09 \%)$ were diagnosed out of 110 patients.

\section{Conclusion}

Pleural effusion of unknown origin hemorrhagic and non-hemorrhagic, are frequently encountered in tertiary care centre even after completion of exhaustive work-up. Fiberoptic bronchoscopy is useful in the 
diagnosis of pleural effusion, where at the end of usual diagnostic work-up, no etiological diagnosis was arrived at. In pleural effusion of unknown origin especially in more than 50 years age group, the contribution of Fiberoptic bronchoscopy in reaching a diagnosis of malignancy is significant. In below 50 years age group with undiagnosed pleural effusion, the contribution of Fiberoptic bronchoscopy in diagnosing nonmalignant causes like Tuberculosis is significant. Hence, In patients with exudative pleural effusion still undiagnosed after pleural fluid cytology and with parenchymal abnormalities on chest skiagram or with history of hemoptysis, Fiberoptic bronchoscopy is a useful procedure for arriving at a diagnosis.

\section{References}

[1]. Chang SC, Perng RP. The role of fiberoptic bronchoscopy in evaluating the causes of pleural effusions. Arch Intern Med 1989;149:855-857.

[2]. Storey DD, Dines DE, Coles DT. Pleural effusion, a diagnostic dilemma.JAMA1976;236:2183-2186.

[3]. Poe RH, Israel RH, Utell MJ, Hall WJ, Greenblatt DW, Kallay MC. Sensitivity, specificity and predictive values of closed pleural biopsy.Arch Intern Med 1984;144:325-328.

[4]. Williams T, Thomas P. The diagnosis of pleural effusion by fiberoptic bronchoscopy and pleuroscopy. Chest 1981;80:566-569

[5]. Lesile WK, Kinasewitz GT. Clinical characteristics of the patient with nonspecific pleuritis. Chest 1988;94:603-608.

[6]. Prakash UBS, Reiman HM. Comparison of needle biopsy with cytologic analysis for the evaluation of pleural effusion: analysis of 414 cases. Mayo Clin Proc 1985;60:158-164.

[7]. Bueno CE, Clemente MG, Castro BC, Martin LM, Ramos SR, Panizo AG, et al. Cytologic and bacteriologic analysis of fluid and pleural biopsy specimens with Cope's needle. Arch Intern Med 1990;150:1190-1194.

[8]. Gunnels JJ: Perplexing pleural effusion. Chest 1978;74:390-393.

[9]. LeRoux BT: Bronchial carcinoma with pleural effusion. S Afr Med J1968;42:865-866.

[10]. Oldenburg FA Jr, Newhouse MT: Thoracoscopy. Chest 1979;75:45-50.

[11]. Meyer PC: Metastatic carcinoma of the pleura. Thorax 1966;221:437-443

[12]. Jarvi OH, Kunnas RJ, Laitio MT, et al: The accuracy and significance of cytologic cancer diagnosis of pleural effusions. Acta Cyto l1972;16:152-158.

[13]. Chernow B,Sahn SA: Carcinomatous Involvement of the pleura. Am J Med 1977;63:695-702. 